\title{
Nongyrotropic particle distributions in space plasmas
}

\author{
U. Motschmann ${ }^{1,2}$, K. H. Glassmeier ${ }^{3}$, A. L. Brinca ${ }^{4}$ \\ ${ }^{1}$ German Aerospace Center (DLR), Institute for Planetary Exploration, D-12484 Berlin, Germany \\ ${ }^{2}$ Now at Institute for Theoretical Physics, Technical University, D-38106 Braunschweig, Germany \\ E-mail: U.Motschmann@tu-bs.de \\ ${ }^{3}$ Institute for Geophysics and Meteorology, Technical University, D-38106 Braunschweig, Germany \\ ${ }^{4}$ Centro de Fisica de Plasmas, Instituto Superior Tecnico, P-1096 Lisboa Codex, Portugal
}

Received: 16 July 1998 / Revised: 10 November 1998 / Accepted: 20 November 1998

\begin{abstract}
In nonstationary, strong inhomogeneous or open plasmas particle orbits are rather complicated. If the nonstationary time scale is smaller than the gyration period, if the inhomogeneity scale is smaller than the gyration radius, i.e. at magnetic plasma boundaries, or if the plasma has sources and sinks in phase space, then nongyrotropic distribution functions occur. The stability of such plasma configurations is studied in the framework of linear dispersion theory. In an open plasma nongyrotropy drives unstable waves parallel and perpendicular to the background magnetic field, whereas in the gyrotropic limit the plasma is stable. In nonstationary plasmas nongyrotropy drives perpendicular unstable waves only. Temporal modulation couples a seed mode with its side lobes and thus it renders unstable wave growth more difficult. As an example of an inhomogeneous plasma a magnetic halfspace is discussed. In a layer with thickness of the thermal proton gyroradius a nongyrotropic distribution is formed which may excite unstable parallel and perpendicular propagating waves.
\end{abstract}

Key words. Interplanetary physics (plasma waves and turbulence) - Ionosphere (plasma waves and instabilities) - Magnetospheric physics (plasma waves and instabilities)

\section{Introduction}

The study of symmetries is an important tool to describe physical phenomena including the equations which are used to model them. This is also true for distribution functions of plasmas where symmetry is mainly discus-

Correspondence to: U. Motschmann sed in the phase space, especially in the velocity space. The state of highest symmetry is isotropy; the distribution function depends only on the value of the velocity vector and surfaces of equal phase space density are spheres. Such distributions usually occur in the absence of any background magnetic fields. In case of a preferred orientation, normally due to an ambient magnetic field, the plasma becomes anisotropic. There is still a symmetry in the plane perpendicular to the magnetic field but in the parallel direction symmetry is broken. Anisotropic plasma distributions are gyrotropic as they are independent of the gyrophase angle. The transition from an isotropic to an anisotropic plasma increases the number of internal degrees of freedom. Consequently the number of possible eigenstates is also increased and expressed by the various wave modes which may exist in such a plasma. If symmetry in the plane perpendicular to the magnetic field is also broken, the distribution function has lost all symmetry properties in the velocity space. It is called nongyrotropic because of the gyrophase dependence is supplemented. The number of degrees of freedom is further increased and new eigenstates are to be expected.

Whereas isotropic and anisotropic plasmas were studied intensively in the last decades widespread interest for nongyrotropic ones has been manifested only since the beginning of the 1990s. These distributions were observed at pickup processes in space plasmas especially when the pickup is burst-like. Evidence for nongyrotropy was found at comets (Neubauer et al., 1993; Glassmeier and Neubauer, 1993; Coates et al., 1993; Cao et al., 1998), at collisionless shocks (Gosling et al., 1982; Anderson et al., 1985; Thomsen et al., 1985; Sckopke et al., 1990; Fuselier et al., 1990), in the plasma environment of the shuttle (Cairns, 1990), at tangential discontinuities in the solar wind (Astudillo et al., 1996), in the plasma sheet boundary layer (Büchner and Kuska, 1996), and in the geomagnetotail (Ashour-Abdalla et al., 1996).

To build up and to sustain a nongyrotropic distribution it is necessary to synchronize the gyrophase angles 
of the particles in any way. This synchronization may be realized in three different ways. One possibility is the existence of sources and sinks which are phase-dependent in velocity space. Inevitably the distribution becomes gyrophase-dependent. Sources and sinks are e.g. ionization and recombination processes. Distributions in such an open phase space may be stationary and homogeneous in space. Another possibility to generate nongyrotropy consists of a temporal modulation of the distribution. An ensemble of particles once bunched in the gyrophase rotates with the gyroperiod and thus forms a nongyrotropic nonstationary distribution. Such a time-dependent nongyrotropy occurs after a burst-like injection of charged particles into the phasespace, e.g. at a pickup process. Groups of particles which are accelerated at a shock or another discontinuity with respect to the plasma background and which are repicked up by the background magnetic field also generate such rotating nongyrotropies. A third way for the generation of a nongyrotropic distribution is provided by inhomogeneous plasmas. Any spatial variation of the magnetic field deforms the circular particle orbits known for homogeneous magnetic fields. In general the orbits may become very complicated or even chaotic (Büchner and Zelenyi, 1989). Thus, a magnetic gradient may bunch the gyrophases and provide a nongyrotropic distribution. A noticable effect, however, is provided only if the inhomogeneity scale is not very large compared with the particle gyroradius. This is especially realized if the plasma inhomogeneity has the structure of a boundary layer.

Nongyrotropic plasmas are not in thermal equilibrium. They carry excess energy and may excite unstable waves. Studies of the stability of rotating nongyrotropic distribution functions and of nongyrotropies maintained by sources and sinks in the phase space were performed by Brinca et al. (1992, 1993), Motschmann and Glassmeier (1993, 1998a) and Cao et al. (1995, 1998). The stability of an inhomogeneous nongyrotropy was discussed for a magnetic reversal by Motschmann and Glassmeier (1998b). In broad ranges of the parameters nongyrotropy contributes to the destabilization of a plasma and to the excitation of unstable wave modes. In many observations the nongyrotropy is superimposed by other sources of free energy such as ring distributions, anisotropy distributions or beams. In these observations it is not always easy to separate the contribution of these different sources. In theoretical studies, however, it is very easy to perform this separation. Pure nongyrotropies without any other source of excess energy were studied by Brinca and Romeiras (1998) and Motschmann and Glassmeier (1998a). In an open phase space and for propagation parallel to the ambient magnetic field they found a destabilization of the right handed mode. For propagation perpendicular to the ambient magnetic field nongyrotropy may excite the extraordinary mode. In a rotating nongyrotropy the temporal modulation of any undisturbed distribution may couple wave modes at frequencies which differ from each other by the gyrofrequency of the nongyrotropic plasma component or its harmonics. Analogous wave mixing at different frequencies is well known in nonlinear optics and high frequency technologies (Schubert and Wilhelmi, 1971). In the excitation process this coupling effects several waves at the same time. Thus, the excess energy is distributed in a broader spectral range and any threshold of the instability is more difficult to hit, as in a quasimonochromatic excitation process. Therefore no instability for parallel propagation was found and for perpendicular propagation unstable wave growth is restricted to narrow parameter ranges.

Nongyrotropic distributions in an inhomogeneous plasma are very difficult to handle. The eigenvalue problem of the stability analysis is not analytically solvable. Even for such intensively studied examples like the Harris sheet (Harris, 1962) only approximate solutions were found (Pritchett et al., 1991; Motschmann and Glassmeier, 1998b). In this study we will discuss a magnetic halfspace which is one of the simplest but nontrivial inhomogeneous configurations. In the transition layer from the field-free halfspace to the magnetic halfspace the ions penetrate into a sheet with a thickness of the order of the thermal gyroradius. In this sheet the ions are phase-bunched and thus they form a nongyrotropic distribution function. This inhomogeneous nongyrotropy may be approached by the open nongyrotropy with sources and sinks in velocity space. Although for the original open nongyrotropy sources and sinks are homogeneously distributed in real space, now sources and sinks are located at the field-free edge of the layer. Thus, as known from open nongyrotropies, unstable waves may be excited parallel and perpendicular to the magnetic field.

The present work is organized in the following way. In Sect. 2 the basic equations are given and the basic types of nongyrotropic distribution functions are discussed. In Sect. 3 an overview of the results of the stability analysis for the different types is given, and in Sect. 4 the results are summarized and conclusions are drawn.

\section{Basic equations}

We consider a plasma consisting of electrons and protons (indices $e$ and $p$ ) with stable distribution functions and an abundance of a nongyrotropic component (index 0) which can be either ions or electrons. The undisturbed nongyrotropic distribution function $F_{0}$ depends on the velocity coordinates $v_{\|}, v_{\perp}$, and especially on the gyrophase angle $\varphi \cdot v_{\|}$and $v_{\perp}$ are velocity components parallel and perpendicular to the stationary background magnetic field $\boldsymbol{B}_{0}=\left(0,0, B_{0}\right)$. The Vlasov equation has the form:

$$
\left(\partial_{t}+\boldsymbol{v} \partial_{x}+\frac{q_{0}}{m_{0}}\left[\boldsymbol{v} \times \boldsymbol{B}_{0}\right] \partial_{v}\right) F_{0}=Q-L .
$$

The Lorenz force term may be simplified and so that

$$
\left(\partial_{t}+v \partial_{x}-\Omega_{0} \partial_{\varphi}\right) F_{0}=Q-L
$$


where the gyrofrequency $\Omega_{0}=q_{0} B_{0} / m_{0}\left(q_{0}\right.$ : charge, $m_{0}$ : mass of the nongyrotropic particles) is introduced. $Q$ and $L$ are source and loss terms, respectively, for the nongyrotropic component. Equation (2) is the starting point for our classification of nongyrotropic plasmas. As the term $\partial_{\varphi} F_{0}$ must not vanish by definition of nongyrotropy it has to be balanced by one of the other terms (or a combination of them). Thus we can distinguish the following basic types.

\subsection{Open nongyrotropy}

The gyrophase dependence is generated by the source $(Q)$ and loss $(L)$ terms which open the velocity space. Therefore this type is abbreviated to open nongyrotropy. This type is of major importance when new plasma ions are generated by ionization processes and when these particles are inserted in the basic plasma by mass-loading. It yields

$-\Omega_{0} \partial_{\varphi} F_{0}=Q-L$.

Integration of Eq. (3) is trivial resulting in

$F_{0}(\varphi)=-\frac{1}{\Omega_{0}} \int_{0}^{\varphi}\left(Q\left(\varphi^{\prime}\right)-L\left(\varphi^{\prime}\right)\right) \mathrm{d} \varphi^{\prime}$.

The arbitrary dependence on $v_{\|}$and $v_{\perp}$ is not indicated here. An example of an open nongyrotropy with source and sink at fixed gyrophases in velocity space $\left(Q \propto \delta\left(\varphi_{Q}\right), L \propto \delta\left(\varphi_{L}\right)\right)$ is sketched in Fig. 1.

\subsection{Rotating nongyrotropy}

When the gyrophase dependence in Eq. (2) is balanced by the time derivative the result is

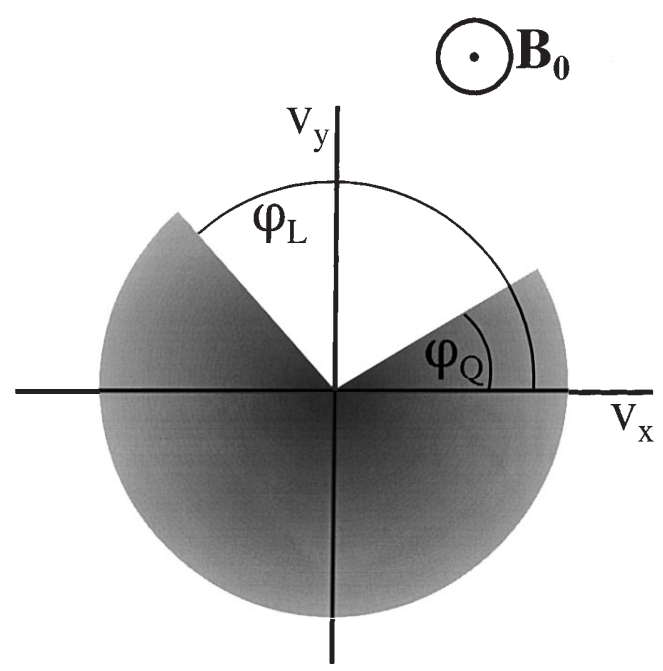

Fig. 1. Sketch of an open nongyrotropic distribution function. Positively charged ions (shaded region) enter the velocity space at $\varphi_{Q}$ and leave it at $\varphi_{L}$
$\left(\partial_{t}-\Omega_{0} \partial_{\varphi}\right) F_{0}=0$.

The solution of Eq. (5) is

$F_{0}=F_{0}\left(\varphi+\Omega_{0} t\right)$.

Thus, the distribution rotates with the gyrofrequency $\Omega_{0}$. An example of such a distribution is sketched in Fig. 2. This type is of importance when ionization processes are finished but the distribution of the inserted particles is still different from the basic plasma distribution.

\subsection{Inhomogeneous nongyrotropy}

When the gyrophase dependence is balanced by the spatial derivative, Eq. (2) provides

$\left(\boldsymbol{v}_{x} \partial_{x}-\Omega_{0} \partial_{\varphi}\right) F_{0}=0$.

Now it is useful to consider an inhomogeneous background magnetic field, that is $\Omega_{0}(x)$. The solution of Eq. (7) depends on the spatial profile of the magnetic field. For a one-dimensional configuration the general solution has the form:

$F_{0}=F_{0}\left(v_{\|}, v_{\perp}, v_{\perp} \sin \varphi+\frac{q_{0}}{m_{0}} A_{y}(x)\right)$,

where the vector potential $A_{y}$ is introduced via $B_{0 z}=\partial_{x} A_{y}$. This type is useful to describe magnetic boundary layers as sketched in Fig. 3 and magnetic reconnection regions (Motschmann and Glassmeier, 1998b).

\section{Stability of nongyrotropic distributions}

Nongyrotropic plasmas are not in thermal equilibrium. They contain excess energy which may drive unstable waves. The excited wave fluctuations provide a

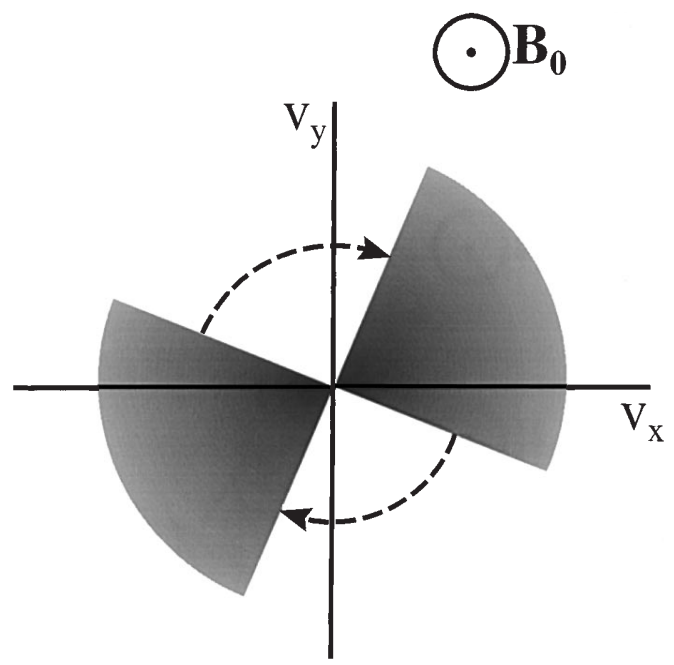

Fig. 2. Sketch of a rotating nongyrotropic distribution function. The whole distribution function of positively charged ions (shaded region) is rotating around the ambient magnetic field. The distribution is symmetric to prevent zero order currents 

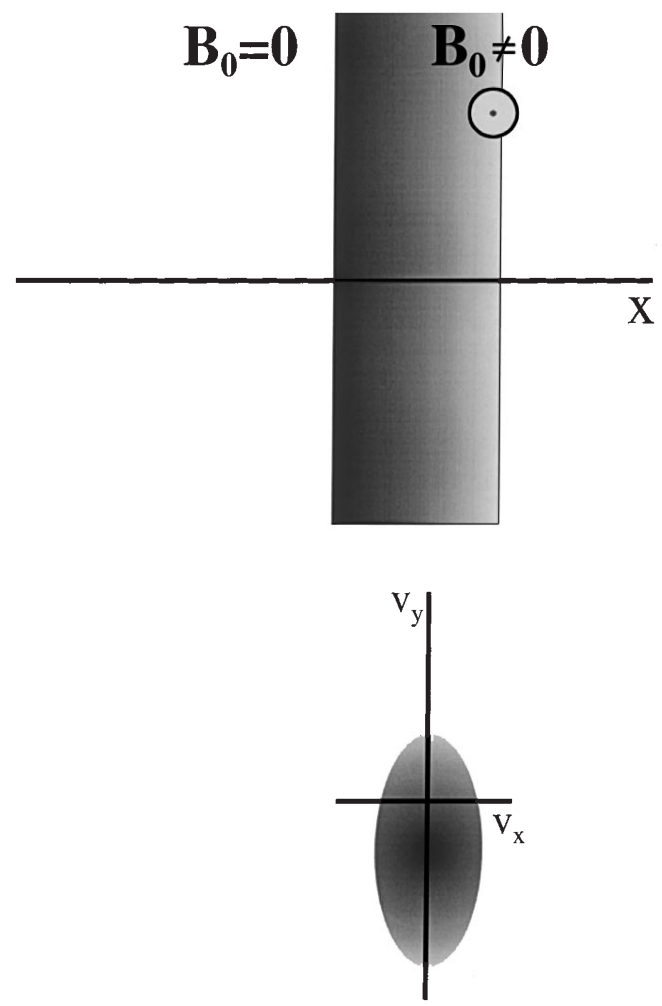

Fig. 3. Sketch of nongyrotropic distribution function in the magnetic boundary layer of a magnetic halfspace

gyrophase diffusion analogous to the pitch angle diffusion. These processes were studied by a particle simulation in earlier work (Kafemann and Motschmann, 1995; Motschmann et al., 1997). A diffusion along the gyrophase angle $\varphi$ exclusively conserving $v_{\perp}$ and $v_{\|}$, however, does not force an instability, as the particle energy $E=m_{0} / 2\left(v_{\|}^{2}+v_{\perp}^{2}\right)$ is independent of $\varphi$. The gyrophase diffusion has to be accompanied e.g. by a decrease of $v_{\|}$to allow transformation of particle energy to wave energy. This synchronous diffusion along $\varphi$ and $v_{\perp}$ may occur if, in the distribution function, underpopulated gyrophase sections exist into which the particles may move with a simultaneous decrease of $v_{\perp}$. A sketch of this diffusion is depicted in Fig. 4.

The stability is studied in the framework of a normal mode analysis. In part we follow a previous paper (Motschmann and Glassmeier, 1998a). The analysis is performed separately for the different types of nongyrotropic distributions where the treatment of the open and the rotating nongyrotropies is similar and both types are presented in one section.

\subsection{Open nongyrotropy and rotating nongyrotropy}

The undisturbed distribution functions (Eq.4 and 6, respectively) may be unified formally by the expression $F_{0}\left(\varphi+\Theta \Omega_{0} t\right)$ where $\Theta=0$ represents the open nongyrotropy and $\Theta=1$ the rotating one. The $2 \pi$ periodicity of $F_{0}$ allows the Fourier expansion into

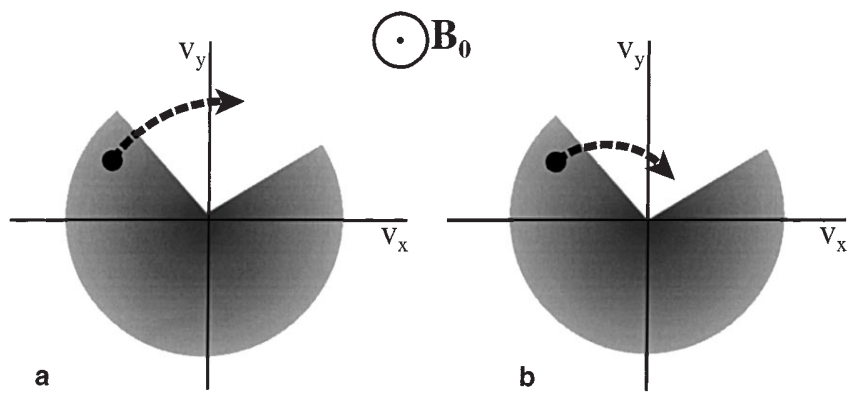

Fig. 4. a,b. Particle diffusion into an undercrowded gyrophase sector. a diffusion process conserving the particle energy; $\mathbf{b}$ diffusion process reducing the particle energy and thus driving an instability

$$
\begin{aligned}
& F_{0}\left(v_{\|}, v_{\perp}, \varphi+\Theta \Omega_{0} t\right) \\
& \quad=\sum_{n=-\infty}^{\infty} F_{0}^{(n)}\left(v_{\|}, v_{\perp}\right) e^{-i n\left(\varphi+\Theta \Omega_{0} t\right)} .
\end{aligned}
$$

The Vlasov equation for the disturbance $F_{1}$ of this distribution $F_{0}$ has the form

$$
\begin{gathered}
{\left[\partial_{\varphi}+i\left(\frac{\boldsymbol{\omega}-k_{\|} v_{\|}}{\Omega_{0}}-\frac{k_{\perp} v_{\perp}}{\Omega_{0}} \cos \varphi\right)\right] F_{1}(\boldsymbol{\omega}, \boldsymbol{k})} \\
=\frac{q_{0}}{m_{0} \Omega_{0}} \sum_{n}\left\{\left[\boldsymbol{E}\left(\boldsymbol{\omega}-\Theta n \Omega_{0}, \boldsymbol{k}\right)\right.\right. \\
\left.\quad+v \times \frac{\boldsymbol{k} \times \boldsymbol{E}\left(\boldsymbol{\omega}-\Theta n \Omega_{0}, \boldsymbol{k}\right)}{\boldsymbol{\omega}-\Theta n \Omega_{0}}\right] \\
\left.\quad \times \partial_{v}\left(F_{0}^{(n)}\left(v_{\|}, v_{\perp}\right) e^{-i n \varphi}\right)\right\}
\end{gathered}
$$

where $\boldsymbol{k}=\left(k_{\perp}, 0, k_{\|}\right)$and $\boldsymbol{v}=\left(v_{\perp} \cos \varphi, v_{\perp} \sin \varphi, v_{\|}\right)$. The wave equation for the (disturbed) electric field $\boldsymbol{E}$ is given by

$$
\left[\left(1-\frac{\boldsymbol{k}^{2} c^{2}}{\omega^{2}}\right) \boldsymbol{I}-\boldsymbol{k} o \boldsymbol{k} \frac{c^{2}}{\omega^{2}}\right] \boldsymbol{E}(\boldsymbol{\omega}, \boldsymbol{k})=-\frac{i}{\epsilon_{0} \omega} \boldsymbol{j}(\boldsymbol{\omega}, \boldsymbol{k}),
$$

with the disturbed current density

$j(\boldsymbol{\omega}, \boldsymbol{k})=n_{0} q_{0} \int v F_{1}\left(\boldsymbol{\omega}, \boldsymbol{k}, v_{\|}, v_{\perp}, \varphi\right) v_{\perp} \mathrm{d} v_{\perp} \mathrm{d} v_{\|} \mathrm{d} \varphi$.

In the following this system of Eqs. (10-12) is solved by standard methods and the conductivity tensor $\boldsymbol{\sigma}$ as well as the dispersion relation are derived. To do this we set $\Theta=0$ in the case of an open nongyrotropy and $\Theta=1$ in the case of a rotating nongyrotropy.

3.1.1. Open nongyrotropy. The conductivity tensor $\sigma$ singled out from Eqs. (10) and (12) is presented in detail in the Appendix. As a standard example a three component plasma consisting of electrons, protons, and alpha particles is discussed. Electrons and protons have stable distribution functions; the nongyrotropic component of alpha particles of density $n_{0}$ is added. The plasma fulfills the neutrality condition $n_{e}=n_{p}+2 n_{0}$. 
For the propagation of waves parallel to the ambient magnetic field $\left(k \| \boldsymbol{B}_{0}\right)$ dispersion relation is used:

$$
\begin{gathered}
\operatorname{det}\left[\left(1-\frac{k_{\|}^{2} c^{2}}{\omega^{2}}\right) \delta_{i j}+\frac{k_{\|}^{2} c^{2}}{\omega^{2}} \delta_{i z}+\frac{i}{\epsilon_{0} \omega} \sum_{e, p, 0} \sigma_{i j}\right]=0 ; \\
i, j \in\{+,-, z\} .
\end{gathered}
$$

In this case it is convenient to use the circularly polarized field amplitudes

$E_{ \pm}=\frac{1}{2}\left(E_{x} \pm i E_{y}\right)$.

First let us remember the equivalent gyrotropic plasma configuration. There are stable right hand polarized $(R)$, left hand polarized $(L)$, and longitudinal polarized $(P)$ modes. The $L$ mode is split near the alpha particle gyroresonance $\Omega_{0}$. Now, in cases where the alpha particles are organized in a nongyrotropic way the $R, L$, and $P$ modes are no longer independent modes but they are coupled with each other. This coupling is expressed by nonvanishing off-diagonal elements of the conductivity tensor $\sigma$. If we restrict to symmetric nongyrotropies, that is $F_{0}(\varphi)=F_{0}(\varphi \pm \pi)$, only $L$ and $R$ modes are coupled (Brinca et al., 1992). The coupling occurs for small $\omega$ and small $k_{\|}$where the phase velocities of both modes are comparable. In the result the $R$ mode becomes unstable and it is excited. Frequency and growth rate are displayed in Fig. 5. For arbitrary (non-symmetric) nongyrotropies Brinca and Romeiras (1998) found an unstable coupling with the $P$ mode too. However, it seems that this instability exists only in a plasma without gyrotropic background as otherwise the damping of the $P$ mode keeps this coupling region stable.

For propagation perpendicular to the magnetic field $\left(\boldsymbol{k} \perp \boldsymbol{B}_{0}\right)$ the ordinary mode is not influenced essentially by the nongyrotropic alpha particles and we focus to the extraordinary mode. Its dispersion relation has the form

$$
\begin{gathered}
\operatorname{det}\left[\delta_{i j}-\frac{k_{\perp}^{2} c^{2}}{\boldsymbol{\omega}^{2}} \delta_{i y}+\frac{i}{\epsilon_{0} \boldsymbol{\omega}} \sum_{e, p, 0} \sigma_{i j}\right]=0 ; \\
i, j \in\{x, y\} .
\end{gathered}
$$

In Eq. (15) the dispersion branches of the higher harmonics of $\Omega_{0}$ and $\Omega_{p}$ are coupled with the fundamental branch complicating its analysis. Therefore we restrict to the limit of long wavelengths $\left(k_{c} v / \Omega_{0}<1\right)$ where the higher harmonics are truncated. Then nongyrotropic alpha particles destabilize the extraordinary mode. Figure 6 shows the maximum growth rate just at the gyroresonance of the alpha particles.

3.1.2. Rotating nongyrotropy. For this basic type the undisturbed distribution function is nonstationary. Thus it is not a standard situation for the stability analysis in the framework of dispersion theory. However, a nonstationary solution of zeroth order may be studied with respect to their stability in the same way as a stationary state; any deviations of the distribution function and the

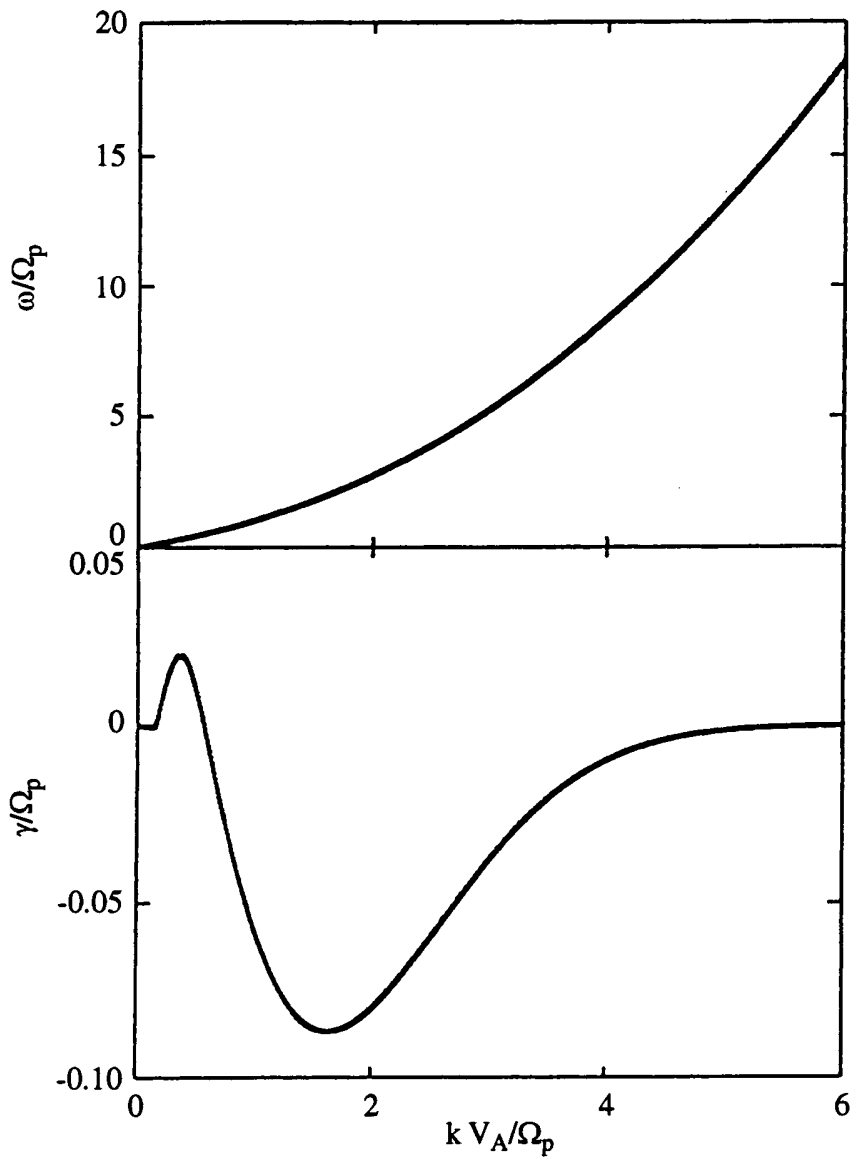

Fig. 5. Parallel propagating unstable mode by $L-R$ coupling forced by an open nongyrotropy of alpha particles embedded in a gyrotropic electron-proton plasma (Motschmann and Glassmeier, 1998a)

fields from the zeroth order have to be checked to see if they are increasing or decreasing.

The rotating nongyrotropy describes a temporal modulation of the undisturbed plasma. From nonlinear optics, high-frequency technology and other fields it is well known that any modulation of a carrier medium forces a frequency mixing of transmitted waves. A wave is scattered by generating side lobes of first and higher orders. The sidelobe distance is just the modulation frequency. In our standard example this is the gyrofrequency of the alpha particles. Thus, any seed field amplitude at $\omega$ is coupled with the seed field amplitudes at $\omega \pm n \Omega_{0}, n=1,2, \ldots$ This coupling is expressed in Ohm's law for the disturbed current $\boldsymbol{j}$ taking now the form

$\boldsymbol{j}(\boldsymbol{\omega}, \boldsymbol{k})=\sum_{n=-\infty}^{\infty} \sigma^{(n)}(\boldsymbol{\omega}, \boldsymbol{k}) \boldsymbol{E}\left(\boldsymbol{\omega}-n \boldsymbol{\Omega}_{0}, \boldsymbol{k}\right)$

The partial conductivity tensors $\sigma^{(n)}$ are given in the Appendix. It is obvious that, in general, the dispersion relation leads us to an infinity dimensional linear system for the $\boldsymbol{E}\left(\boldsymbol{\omega}-n \Omega_{0}\right), n=0, \pm 1, \pm 2, \ldots$ In practice it is to be expected that the side lobes are restricted to a few orders. Thus higher orders can be truncated dependent on the respective example. 


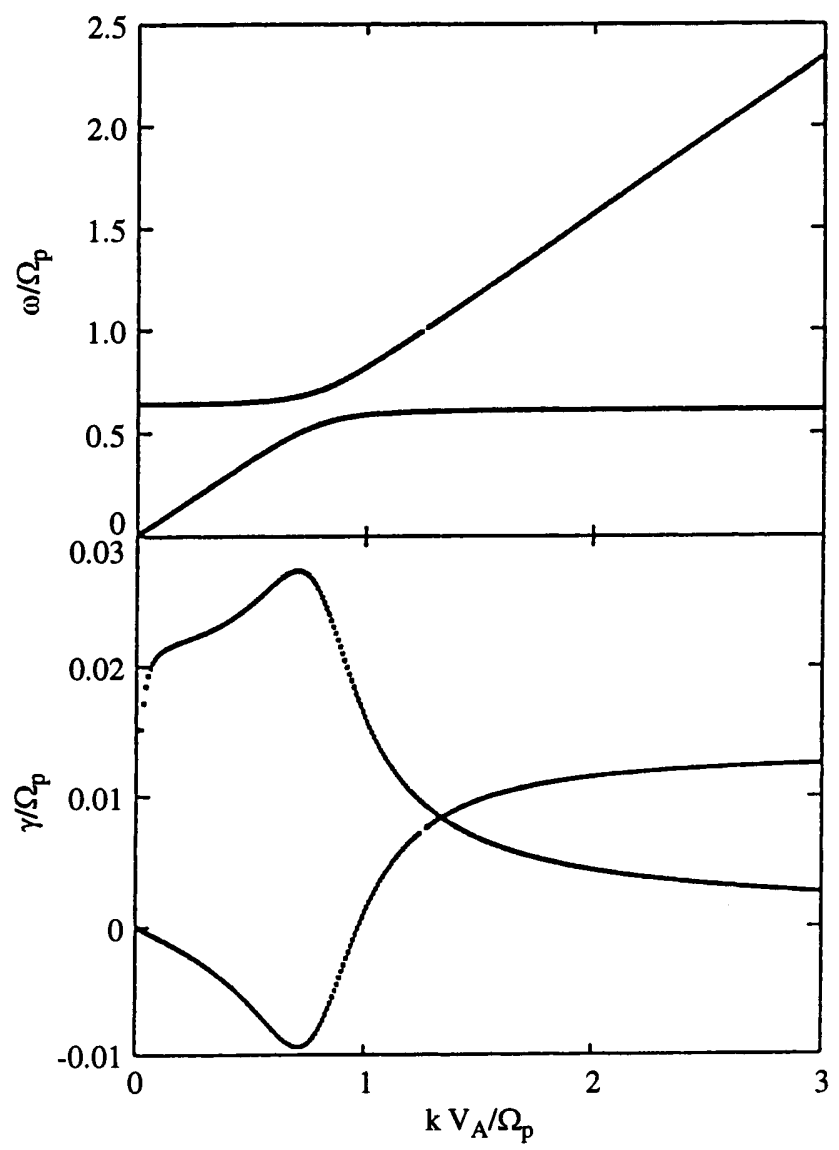

Fig. 6. Perpendicular propagating unstable extraordinary mode forced by an open nongyrotropy of alpha particles embedded in a gyrotropic electron-proton plasma (Motschmann and Glassmeier, 1998a)

In the special case of parallel propagation $\left(\boldsymbol{k} \| \boldsymbol{B}_{0}\right)$ the coupling truncates exactly at the second order. All partial conductivity tensors $\sigma^{(n)}$ with $|n|>2$ vanish. We found that for this case nongyrotropy does not excite any unstable waves (Motschmann and Glassmeier, 1998a). The coupling takes place, but the available excess energy is distributed over a broad spectral range and the threshold for an instability is not reached. We mention that an additional energy contribution, e.g. by an anisotropy, will indeed excite unstable waves as discussed by Brinca et al. (1992) and Motschmann and Glassmeier (1993). In this plasma the instability is mainly driven by the anisotropy; the coupling of wave amplitudes up to the side lobes of the second order caused by the nongyrotropy is maintained.

At perpendicular propagation $\left(\boldsymbol{k} \perp \boldsymbol{B}_{0}\right)$ the coupling does not truncate automatically at higher orders. We consider the long wavelength approximation analogous to the open nongyrotropy where coupling is restricted to first order side lobes (Motschmann and Glassmeier, 1998a) . Thus, the excess energy is bundled in narrow spectral region. In this case a strong instability is excited. Frequency and growth rate are shown in Fig. 7. Maximum growth rate is reached at frequencies in between the gyrofrequency $\Omega_{0}$ of the alpha particles and the side lobe at $2 \Omega_{0}$. Studies of short wavelengths $\left(k_{\perp} \Omega_{0} / v_{A} \geq 1\right)$ do not exist yet. We speculate that no instability or only a weak version will be excited as the excess energy would be distributed over a broader spectral range.

\subsection{Inhomogeneous nongyrotropy}

In an inhomogeneous magnetic field the particle orbits deviate from circular ones. In general they may become rather complicated and even chaotic (Ashour-Abdalla et al., 1991). Then the distribution functions are gyrophase-dependent too, that is nongyrotropic. The detailed shape depends on the spatial profile of the background magnetic field. Strong gyrophase-dependence is provided in cases where the spatial scale of the inhomogeneity is at least of the same order as the thermal gyroradius. Because of the smallness of this scale it is more convenient to speak of a boundary layer than of a inhomogeneous space.

An analytic handling of an inhomogeneous nongyrotropy is not trivial. Therefore we discuss as a fairly simple example a magnetic halfspace as sketched in Fig. 3 and we regard this example as a prototype of real magnetic boundaries. However, even this example is not solvable in a fully exact way and thus we perform some approximations. The transition from the field-free

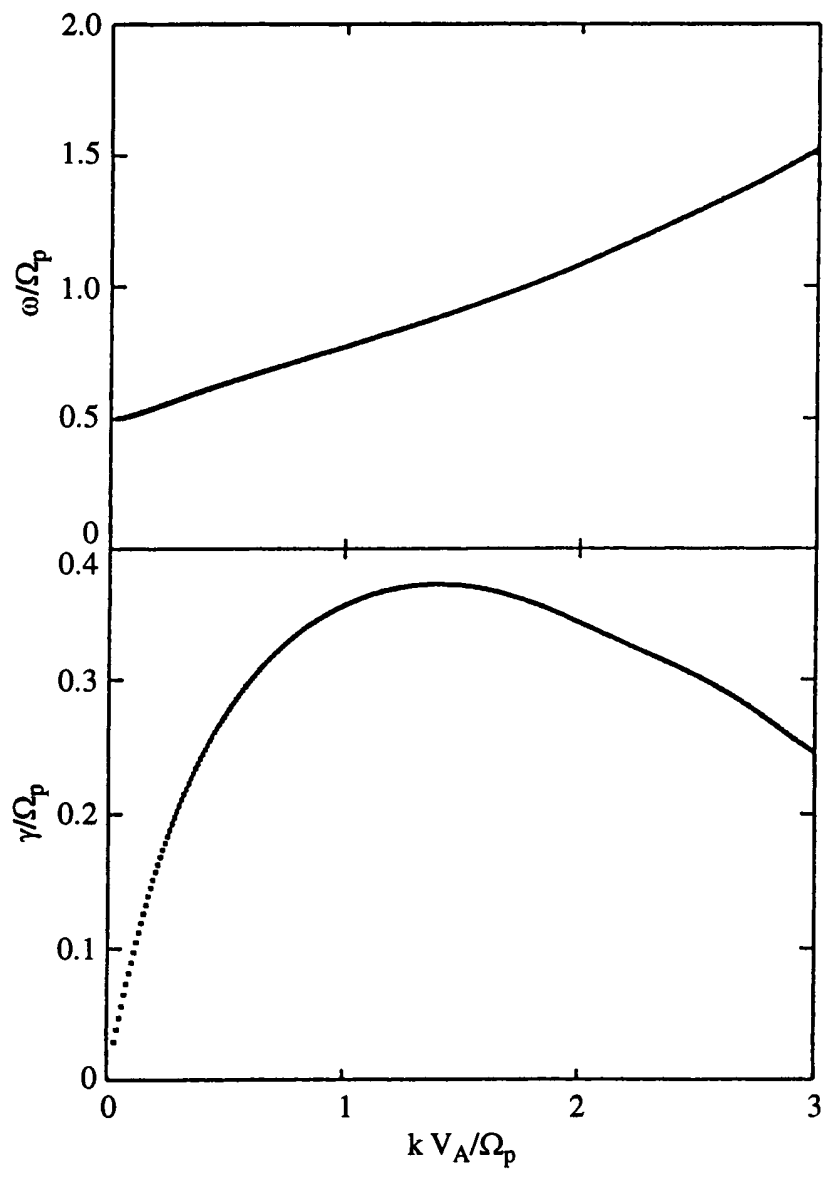

Fig. 7. Perpendicular propagating unstable extra ordinary mode forced by a rotating nongyrotropy (Motschmann and Glassmeier, 1998a) 
region to the magnetic region is assumed to be sharp such that the characteristic gradient scale is small compared with the gyroradii of the particles. An electron-proton plasma as discussed here forms a nongyrotropic sheet just at the edge of the magnetic halfspace. The thickness of the sheet is of the order of the proton gyroradius. As the electron gyroradius is much smaller we do not take their nongyrotropy into account.

For the magnetic halfspace the vector potential $A_{y}$ yields

$A_{y}=\left\{\begin{array}{ccc}0 & \text { for } & x<0 \\ B_{z} x & \text { for } & x>0\end{array}\right\}$.

For the construction of the undisturbed nongyrotropic distribution function we refer to the general solution (8) of a one-dimensional inhomogeneity and choose the shape of the form

$$
\begin{aligned}
& F_{0}\left(v_{\|}, v_{\perp}, \varphi, x\right) \\
& \quad=\left\{\begin{array}{ccc}
0 & \text { for } & x<0 \\
\frac{1}{\left(\sqrt{\pi} v_{t p}\right)^{3}} e^{-\frac{v_{\|}^{2}+v_{\perp}^{2}}{v_{t p}^{2}}} e^{\frac{v_{\perp} \sin \varphi}{v_{t p}}} e^{-\frac{x}{r_{t p}}} & \text { for } & x>0
\end{array}\right\} .
\end{aligned}
$$

This $\varphi$ dependent distribution $F_{0}$ is restricted to the transition layer from the field-free halfspace to the magnetic halfspace. The protons which form this distribution we regard as a third component embedded in a gyrotropic electron-proton plasma. $v_{t p}$ is thermal proton velocity and $r_{t p}=v_{t p} / \Omega_{p}$ is the corresponding gyroradius. To keep the further analysis tractable we will approach expression (18) by a rectangular shape of the form

$$
\begin{aligned}
& F_{0}\left(v_{\|}, v_{\perp}, \varphi, x\right) \\
& \quad=\left\{\begin{array}{cc}
0 & \text { for } \quad x<0 \text { and } x>r_{t p} \\
\frac{1}{\left(\sqrt{\pi} v_{t p}\right)^{3}} e^{-\frac{v_{\|}^{2}+v_{\perp}^{2}}{v_{t p}^{2}}} e^{-\frac{v_{\perp} \sin \varphi}{v_{t p}}} & \text { for } \quad 0<x<r_{t p}
\end{array}\right\} .
\end{aligned}
$$

This distribution (19) may be expanded in a Fourier series analogous to expression (9) for an open nongyrotropy $\Theta=0$. However, there is not only a formal equivalence of the open and the inhomogeneous nongyrotropies. In Sect. 3.1 we assumed sources and sinks in the velocity space to produce a gyrophase dependence. Now we replace this by sources and sinks in real space. Indeed the sources and sinks for the nongyrotropy are located at $x=0$. Thus we can take over the results of Sect. 3.1 for an open nongyrotropy. In particular, we conclude that the distribution (19) is unstable with respect to parallel and perpendicular wave propagation where perpendicular propagation is now taken along $y$. To avoid a conflict when applying the results of the open nongyrotropy in free space to the layer we restrict our discussion to wavelengths shorter than the thickness $r_{p t}$ of the layer. Unstable waves fulfill $k v_{A} / \Omega_{p} \sim I$ as is seen in Fig. 5. This corresponds to a wavelength of $\lambda \sim 2 \pi r_{p t} v_{A} / v_{t p} \sim 2 \pi r_{p i} \sqrt{\beta}_{p t}$. Thus for a high proton plasma beta $\left(\beta_{p} \gg I\right)$ the result is $\lambda \ll r_{t p}$ and our model is consistent.

\section{Summary and conclusions}

The gyrophase-dependence of a distribution function introduces new degrees of freedom in the wave-particle system of a collisionless plasma. A classification of the various types of nongyrotropic distribution functions is performed. Three basic types may be discriminated: the nongyrotropy caused by sources and sinks in velocity space, the rotating nongyrotropy, and nongyrotropy because of an inhomogeneity of the ambient magnetic field. These nongyrotropic distributions are not in thermal equilibrium. They carry excess energy which may excite unstable waves. The stability of the basic types were discussed in the framework of linear dispersion analysis. All types are unstable for convenient ranges of the plasma parameters.

Open nongyrotropies may drive waves parallel as well as perpendicular to the background magnetic field. At parallel propagation and at symmetric nongyrotropies the $R$-mode and the $L$-mode are coupled where the $R$-mode is destabilized. At perpendicular propagation the extraordinary mode is destabilized where the growth rate becomes maximum at the gyrofrequency of the nongyrotropic plasma component.

The rotating nongyrotropy couples a seed wave with its side lobes caused by the temporal modulation of the undisturbed plasma. This coupling has the tendency to stabilize the system as the excess energy is smeared out by means of the side lobes over broad spectral range but not transformed to a wave quasimonochromatically. Therefore at parallel propagation any instability is suppressed. At perpendicular propagation a strong instability is excited in the long wavelength limit where the coupling is restricted to side lobes of the first order.

The inhomogeneous nongyrotropy has been studied for the case of the magnetic halfspace. In the transition layer from the field-free region to the magnetic region a nongyrotropic distribution is generated because of incomplete circular particle orbits. The distribution in the layer has the thickness of the order of the thermal ion gyration radius. It may be approached by the open nongyrotropic type, where spatially distributed sources and sinks are replaced now by sources and sinks spatially localized at the edge of the layer. In general this nongyrotropic distribution in the layer is unstable and it may excite parallel and perpendicular waves. We speculate that this process in its nonlinear stage may reorganize the original layer structure.

Acknowledgements. The work of U.M. is supported by the Deutsche Forschungsgemeinschaft through a Werner-HeisenbergStipendium. The work of K.H.G. is financially supported by DLR. The authors thank the members of the Nongyrotropy in Space Plasmas project for useful discussions. 
The Editor-in-chief thanks N. Watkins and another referee for their help in evaluating this paper.

\section{Appendix}

In case of the open nongyrotropy $\Theta=0$ where the undisturbed plasma is assumed to be stationary and homogeneous Ohm's law has the conventional form

$$
\boldsymbol{j}(\omega, \boldsymbol{k})=\boldsymbol{\sigma}(\omega, \boldsymbol{k}) E(\omega, \boldsymbol{k})
$$

From Eqs. (10) and (12) the conductivity tensor $\boldsymbol{\sigma}$ can be singled out. It yields

$$
\begin{aligned}
& \sigma_{++}=\frac{i \pi n_{0} q_{0}^{2}}{m_{0}} \sum_{n, l} \int v_{\perp}^{2} \mathrm{~d} v_{\perp} \mathrm{d} v_{\|} \\
& \times\left[\frac{J_{n+l} J_{l}}{k_{\|} v_{\|}-\omega+(n+l+1) \Omega_{0}} g_{+}^{(n)}\right. \\
& \left.+\frac{J_{n+l-1} J_{l}}{k_{\|} v_{\|}-\omega+(n+l) \Omega_{0}} c_{+}^{(n)}\right] \\
& \sigma_{+-}=\frac{i \pi n_{0} q_{0}^{2}}{m_{0}} \sum_{n, l} \int v_{\perp}^{2} \mathrm{~d} v_{\perp} \mathrm{d} v_{\|} \\
& \times\left[\frac{J_{n+l-1} J_{l}}{k_{\|} v_{\|}-\omega+(n+l) \Omega_{0}} c_{-}^{(n)}\right. \\
& \left.+\frac{J_{n+l-2} J_{l}}{k_{\|} v_{\|}-\omega+(n+l-1) \Omega_{0}} g_{-}^{(n)}\right] \\
& \sigma_{+z}=\frac{i \pi n_{0} q_{0}^{2}}{m_{0}} \sum_{n, l} \int v_{\perp}^{2} \mathrm{~d} v_{\perp} \mathrm{d} v_{\|} \\
& \times\left[\frac{J_{n+l} J_{l}}{k_{\|} v_{\|}-\omega+(n+l+1) \Omega_{0}} h_{+}^{(n)}\right. \\
& +\frac{J_{n+l-1} J_{l}}{k_{\|} v_{\|}-\omega+(n+l) \Omega_{0}} f^{(n)} \\
& \left.+\frac{J_{n+l-2} J_{l}}{k_{\|} v_{\|}-\omega+(n+l-1) \Omega_{0}} h_{-}^{(n)}\right] \\
& \sigma_{-+}=\frac{i \pi n_{0} q_{0}^{2}}{m_{0}} \sum_{n, l} \int v_{\perp}^{2} \mathrm{~d} v_{\perp} \mathrm{d} v_{\|} \\
& \times\left[\frac{J_{n+l+2} J_{l}}{k_{\|} v_{\|}-\omega+(n+l+1) \Omega_{0}} g_{+}^{(n)}\right. \\
& \left.+\frac{J_{n+l+1} J_{l}}{k_{\|} v_{\|}-\omega+(n+l) \Omega_{0}} c_{+}^{(n)}\right] \\
& \sigma_{--}=\frac{i \pi n_{0} q_{0}^{2}}{m_{0}} \sum_{n, l} \int v_{\perp}^{2} \mathrm{~d} v_{\perp} \mathrm{d} v_{\|} \\
& \times\left[\frac{J_{n+l+1} J_{l}}{k_{\|} v_{\|}-\omega+(n+l) \Omega_{0}} c_{-}^{(n)}\right. \\
& \left.+\frac{J_{n+l} J_{l}}{k_{\|} v_{\|}-\omega+(n+l-1) \Omega_{0}} g_{-}^{(n)}\right]
\end{aligned}
$$

$$
\begin{aligned}
& \sigma_{-z}=\frac{i \pi n_{0} q_{0}^{2}}{m_{0}} \sum_{n, l} \int v_{\perp}^{2} \mathrm{~d} v_{\perp} \mathrm{d} v_{\|} \\
& \times\left[\frac{J_{n+l+2} J_{l}}{k_{\|} v_{\|}-\omega+(n+l+1) \Omega_{0}} h_{+}^{(n)}\right. \\
& +\frac{J_{n+l+1} J_{l}}{k_{\|} v_{\|}-\omega+(n+l) \Omega_{0}} f^{(n)} \\
& \left.+\frac{J_{n+l} J_{l}}{k_{\|} v_{\|}-\omega+(n+l-1) \Omega_{0}} h_{-}^{(n)}\right] \\
& \sigma_{z+}=\frac{i \pi n_{0} q_{0}^{2}}{m_{0}} \sum_{n, l} \int v_{\perp} \mathrm{d} v_{\perp} v_{\|} \mathrm{d} v_{\|} \\
& \times\left[\frac{J_{n+l+1} J_{l}}{k_{\|} v_{\|}-\omega+(n+l+1) \Omega_{0}} g_{+}^{(n)}\right. \\
& \left.+\frac{J_{n+l} J_{l}}{k_{\|} v_{\|}-\omega+(n+l) \Omega_{0}} c_{+}^{(n)}\right] \\
& \sigma_{z-}=\frac{i \pi n_{0} q_{0}^{2}}{m_{0}} \sum_{n, l} \int v_{\perp} \mathrm{d} v_{\perp} v_{\|} \mathrm{d} v_{\|} \\
& \times\left[\frac{J_{n+l} J_{l}}{k_{\|} v_{\|}-\omega+(n+l) \Omega_{0}} c_{-}^{(n)}\right. \\
& \left.+\frac{J_{n+l-1} J_{l}}{k_{\|} v_{\|}-\omega+(n+l-1) \Omega_{0}} g_{-}^{(n)}\right] \\
& \sigma_{z z}=\frac{i \pi n_{0} q_{0}^{2}}{m_{0}} \sum_{n, l} \int v_{\perp} \mathrm{d} v_{\perp} v_{\|} \mathrm{d} v_{\|} \\
& \times\left[\frac{J_{n+l+1} J_{l}}{k_{\|} v_{\|}-\omega+(n+l+1) \Omega_{0}} h_{+}^{(n)} .\right. \\
& +\frac{J_{n+l} J_{l}}{k_{\|} v_{\|}-\omega+(n+l) \Omega_{0}} f^{(n)} \\
& \left.+\frac{J_{n+l-1} J_{l}}{k_{\|} v_{\|}-\omega+(n+l-1) \Omega_{0}} h_{-}^{(n)}\right]
\end{aligned}
$$

where

$$
\begin{aligned}
& c_{ \pm}^{(n)}= \pm \frac{k_{\perp}}{\omega} n F_{0}^{(n)}, \quad f^{(n)}=\partial_{v_{\|}} F_{0}^{(n)} \\
& g_{ \pm}^{(n)}=\left[\frac{k_{\|} v_{\perp}}{\omega} \partial_{v_{\|}}+\left(1-\frac{k_{\|} v_{\|}}{\omega}\right)\left(\partial_{v_{\perp}} \mp \frac{n}{v_{\perp}}\right)\right] F_{0}^{(n)} \\
& h_{ \pm}^{(n)}=\frac{1}{2}\left[-\frac{k_{\perp} v_{\perp}}{\omega} \partial_{v_{\|}}+\frac{k_{\perp} v_{\|}}{\omega}\left(\partial_{v_{\perp}} \mp \frac{n}{v_{\perp}}\right)\right] F_{0}^{(n)} .
\end{aligned}
$$

The argument of the Bessel functions $J_{l}$ is $k_{\perp} v / \Omega_{0}$.

For the rotating nongyrotropy $(\Theta=1)$ Ohm's law has the form given by Eqn. (16). The partial conductivity tensors $\sigma^{(n)}$ analogous to the open nongyrotropy is produced from Eqs. (10) and (12). It follows:

$$
\begin{aligned}
\sigma_{++}^{(n)}= & \frac{i \pi n_{0} q_{0}^{2}}{m_{0}} \sum_{l} \int v_{\perp}^{2} \mathrm{~d} v_{\perp} \mathrm{d} v_{\|} \\
& \times\left[\frac{J_{n+l} J_{l}}{k_{\|} v_{\|}-\omega+(n+l+1) \Omega_{0}} g_{+}^{(n)}\right.
\end{aligned}
$$




$$
\begin{aligned}
& \left.+\frac{J_{n+l-1} J_{l}}{k_{\|} v_{\|}-\omega+(n+l) \Omega_{0}} c_{+}^{(n)}\right] \\
& \sigma_{+-}^{(n)}=\frac{i \pi n_{0} q_{0}^{2}}{m_{0}} \sum_{l} \int v_{\perp}^{2} \mathrm{~d} v_{\perp} \mathrm{d} v_{\|} \\
& \times\left[\frac{J_{n+l-1} J_{l}}{k_{\|} v_{\|}-\omega+(n+l) \Omega_{0}} c_{-}^{(n)}\right. \\
& \left.+\frac{J_{n+l-2} J_{l}}{k_{\|} v_{\|}-\omega+(n+l-1) \Omega_{0}} g_{-}^{(n)}\right] \\
& \sigma_{+z}^{(n)}=\frac{i \pi n_{0} q_{0}^{2}}{m_{0}} \sum_{l} \int v_{\perp}^{2} \mathrm{~d} v_{\perp} \mathrm{d} v_{\|} \\
& \times\left[\frac{J_{n+l} J_{l}}{k_{\|} v_{\|}-\omega+(n+l+1) \Omega_{0}} h_{+}^{(n)}\right. \\
& +\frac{J_{n+l-1} J_{l}}{k_{\|} v_{\|}-\omega+(n+l) \Omega_{0}} f^{(n)} \\
& \left.+\frac{J_{n+l-2} J_{l}}{k_{\|} v_{\|}-\omega+(n+l-1) \Omega_{0}} h_{-}^{(n)}\right] \\
& \sigma_{-+}^{(n)}=\frac{i \pi n_{0} q_{0}^{2}}{m_{0}} \sum_{l} \int v_{\perp}^{2} \mathrm{~d} v_{\perp} \mathrm{d} v_{\|} \\
& \times\left[\frac{J_{n+l+2} J_{l}}{k_{\|} v_{\|}-\omega+(n+l+1) \Omega_{0}} g_{+}^{(n)}\right. \\
& \left.+\frac{J_{n+l+1} J_{l}}{k_{\|} v_{\|}-\omega+(n+l) \Omega_{0}} c_{+}^{(n)}\right] \\
& \sigma_{--}^{(n)}=\frac{i \pi n_{0} q_{0}^{2}}{m_{0}} \sum_{l} \int v_{\perp}^{2} \mathrm{~d} v_{\perp} \mathrm{d} v_{\|} \\
& \times\left[\frac{J_{n+l+1} J_{l}}{k_{\|} v_{\|}-\omega+(n+l) \Omega_{0}} c_{-}^{(n)}\right. \\
& \left.+\frac{J_{n+l} J_{l}}{k_{\|} v_{\|}-\omega+(n+l-1) \Omega_{0}} g_{-}^{(n)}\right] \\
& \sigma_{-z}^{(n)}=\frac{i \pi n_{0} q_{0}^{2}}{m_{0}} \sum_{l} \int v_{\perp}^{2} \mathrm{~d} v_{\perp} \mathrm{d} v_{\|} \\
& \times\left[\frac{J_{n+l+2} J_{l}}{k_{\|} v_{\|}-\omega+(n+l+1) \Omega_{0}} h_{+}^{(n)}\right. \\
& +\frac{J_{n+l+1} J_{l}}{k_{\|} v_{\|}-\omega+(n+l) \Omega_{0}} f^{(n)} \\
& \left.+\frac{J_{n+l} J_{l}}{k_{\|} v_{\|}-\omega+(n+l-1) \Omega_{0}} h_{-}^{(n)}\right] \\
& \sigma_{z+}^{(n)}=\frac{i \pi n_{0} q_{0}^{2}}{m_{0}} \sum_{l} \int v_{\perp} \mathrm{d} v_{\perp} v_{\|} \mathrm{d} v_{\|} \\
& \times\left[\frac{J_{n+l+1} J_{l}}{k_{\|} v_{\|}-\omega+(n+l+1) \Omega_{0}} g_{+}^{(n)}\right. \\
& \left.+\frac{J_{n+l} J_{l}}{k_{\|} v_{\|}-\omega+(n+l) \Omega_{0}} c_{+}^{(n)}\right] \\
& \sigma_{z-}^{(n)}=\frac{i \pi n_{0} q_{0}^{2}}{m_{0}} \sum_{l} \int v_{\perp} \mathrm{d} v_{\perp} v_{\|} \mathrm{d} v_{\|}
\end{aligned}
$$

$$
\begin{aligned}
& \times\left[\frac{J_{n+l} J_{l}}{k_{\|} v_{\|}-\omega+(n+l) \Omega_{0}} c_{-}^{(n)}\right. \\
& \left.+\frac{J_{n+l-1} J_{l}}{k_{\|} v_{\|}-\omega+(n+l-1) \Omega_{0}} g_{-}^{(n)}\right] \\
\sigma_{z z}^{(n)}= & \frac{i \pi n_{0} q_{0}^{2}}{m_{0}} \sum_{l} \int v_{\perp} \mathrm{d} v_{\perp} v_{\|} \mathrm{d} v_{\|} \\
& \times\left[\frac{J_{n+l+1} J_{l}}{k_{\|} v_{\|}-\omega+(n+l+1) \Omega_{0}} h_{+}^{(n)}\right. \\
& +\frac{J_{n+l} J_{l}}{k_{\|} v_{\|}-\omega+(n+l) \Omega_{0}} f^{(n)} \\
& \left.+\frac{J_{n+l-1} J_{l}}{k_{\|} v_{\|}-\omega+(n+l-1) \Omega_{0}} h_{-}^{(n)}\right]
\end{aligned}
$$

where now,

$$
\begin{aligned}
& c_{ \pm}^{(n)}= \pm \frac{k_{\perp}}{\omega-n \Omega_{0}} n F_{0}^{(n)}, \quad f^{(n)}=\partial_{v_{\|}} F_{0}^{(n)} \\
& g_{ \pm}^{(n)}=\left[\frac{k_{\|} v_{\perp}}{\omega-n \Omega_{0}} \partial_{v_{\|}}+\left(1-\frac{k_{\|} v_{\|}}{\omega-n \Omega_{0}}\right)\left(\partial_{v_{\perp}} \mp \frac{n}{v_{\perp}}\right)\right] F_{0}^{(n)} \\
& h_{ \pm}^{(n)}=\frac{1}{2}\left[-\frac{k_{\perp} v_{\perp}}{\omega-n \Omega_{0}} \partial_{v_{\|}}+\frac{k_{\perp} v_{\|}}{\omega-n \Omega_{0}}\left(\partial_{v_{\perp}} \mp \frac{n}{v_{\perp}}\right)\right] F_{0}^{(n)} .
\end{aligned}
$$

\section{References}

Anderson, K. A., R. P. Lin, C. Gurgiolo, G. K. Parks, D. W. Potter, S. Werden, and H. Reme, A component of nongyrotropic (phase-bunched) electrons upstream from the Earth's bow shock, J. Geophys. Res., 90, 10 809-10 814, 1985.

Ashour-Abdalla, M., L. M. Zelenyi, V. Peroomian, L. A. Frank, and W. R. Paterson, Coarse-grained texture of ion distributions in the magnetotail: a fractal-like approach, J. Geophys. Res., 101, 15 287-15 296, 1996.

Ashour-Abdalla, M., J. Büchner, and L. M. Zelenyi, The quasiadiabatic ion distribution in the central plasma sheet and its boundary layer, J. Geophys. Res., 96, 1601-1609, 1991.

Astudillo, H. F., S. Livi, E. Marsch and H. Rosenbauer, Evidence for nongyrotropic alpha particle and proton distribution functions: TAUS solar wind measurements, J. Geophys. Res., 101, 24 423-24 432, 1996.

Brinca, A. L., and F. J. Romeiras, On the stability of stationary nongyrotropic distribution functions: coupling and purely growing waves, J. Geophys. Res., 103, 9275-9283, 1998.

Brinca, A. L., L. Borda de Água, and D. Winske, Nongyrotropy as a source of instability and mode coupling, Geophys. Res. Lett., 12, 2445-2448, 1992.

Brinca, A. L., Y. Omura, and H. Matsumoto, Linear coupling effects originated in electron nongyrotropy, J. Geophys. Res., 98, 21 071-21 076, 1993.

Büchner, J., and J. P. Kuska, On the formation of cup-like ion beam distributions in the plasma sheet boundary layer, $J$. Geomagn. Geoelectr., 48, 781-797, 1996.

Büchner, J., and L. M. Zelenyi, Regular and chaotic charged particle motion in magnetotaillike field reversals, 1 . Basic theory of trapped motion, J. Geophys. Res., 94, 11 821-11 842, 1989.

Cairns, I. H., Transition from ring to beam arc distributions of water ions near the space shuttle orbiter, J. Geophys. Res., 95, 15 167-15 173, 1990

Cao, J. B., C. Mazelle, G. Belmont, and H. Reme, The nongyrotropy of heavy newborn ions at comet Grigg-Skjellerup and 
corresponding instability, J. Geophys. Res., 100, 23 379-23 388, 1995.

Cao, J. B., C. Mazelle, G. Belmont, and H. Reme, Oblique ring instability driven by nongyrotropic ions: application to observations at comet Grigg-Skjellerup, J. Geophys. Res., 103, 2055, 1998.

Coates, A. J., A. D. Johnstone, B. Wilken, and F. M. Neubauer, Velocity space diffusion and nongyrotropy of pickup water group ions at comet Grigg-Skjellerup, J. Geophys. Res., 98, 20 985-20 994, 1993.

Fuselier, S. A., O. W. Lennartsson, M. F. Thomsen, C. T. Russell, Specularly reflected $\mathrm{He}^{2+}$ at high Mach number quasi-parallel shocks, J. Geophys. Res., 95, 4319-4325, 1990.

Glassmeier, K. H., and F. M. Neubauer, Low-frequency electromagnetic plasma waves at comet $\mathrm{P} /$ Grigg-Skjellerup: overview and spectral characteristics, J. Geophys. Res., 98, 20 921, 1993.

Gosling, J. T., M. F. Thomsen, S. J. Bame, W. C. Feldman, G. Paschmann, and N. Sckopke, Evidence for specularly reflected ions upstream from the quasi-parallel bow shock, Geophys. Res. Lett., 9, 1333-1336, 1982.

Harris, E. G., On a plasma sheath sparating regions of oppositely directed magnetic field, Il Nuovo Cimento, XXIII, 115-121, 1962.

Kafemann, H., and U. Motschmann, Studies on nongyrotropic ion distributions in magneto plasmas by 2 -d hybrid code simulation, Simpo Newsl. 5, 14-19, 1995.

Motschmann, U., and K. H. Glassmeier, Nongyrotropic distribution of pickup ions at comet $\mathrm{P} /$ Grigg-Skjellerup: a possible source of wave activity, J. Geophys. Res., 98, 20 977-20 983, 1993.
Motschmann, U., H. Kafemann, and M. Scholer, Nongyrotropy in magnetoplasmas: Simulation of wave excitation and phase space diffusion, Ann. Geophysicae, 15, 603-613, 1997.

Motschmann U., and K. H. Glassmeier, Dispersion and wave excitation in nongyrotropic plasmas, J. Plasma Phys., 60, 111132, 1998a.

Motschmann U., and K. U. Glassmeier, Relation of magnetic field line reconnection and unstable nongyrotropic particle distributions, Proc. Int. Conf. on Substorms-4, Lake Hamana, Japan, in press, $1998 \mathrm{~b}$

Neubauer, F. M., K. H. Glassmeier, A. J. Coates, and A. D. Johnston, Low frequency elctromagnetic plasma waves at comet P/Grigg-Skjellerup: analysis and interpretation, J. Geophys. Res., 98, 20 937-20 953, 1993.

Pritchett, P. L., F. V. Coroniti, and R. Pellat, Collisionless reconnection in two-dimensional magnetotail equilibria, J. Geophys. Res., 96, 11 523-11 538, 1991.

Schubert, M., and B. Wilhelmi, Einführung in die nichtlineare Optik, Teubner Verlagsgesellschaft, Leipzig, 1971.

Sckopke, N., G. Paschmann, A. L. Brinca, C. W. Carlson, and H. Lühr, Ion thermalisation in quasi-perpendicular shocks involving reflected ions, J. Geophys. Res., 95, 6337-6352, 1990.

Thomsen, M. F., J. T. Gosling, S. J. Bame, and C. T. Russell, Gyrating ions and large-amplitude monochromatic MHD waves upstream of the Earth's bow shock, J. Geophys. Res., 90, 267-273, 1985. 